\title{
Studies of Combustion Reactions at the \\ State-Resolved Differential Cross Section Level
}

Progress Report

for Period July 1, 1991 - February 28, 19y2.

Paul L. Houston

Department of Chemistry

Cornell University

Ithaca, NY 14853-1301

March 25, 1992

Prepared for

THE U. S. DEPARTMENT OF ENERGY

AGREEMENT NO. DE-FG02-88ER 13934 


\section{Abstract}

State-resolved differential reaction cross sections provide perhaps the most detailed information about the mechanism of a chemical reaction, but heretofore they have been extremely difficult to measure. This program explores a new techrique for obtaining differential cross sections with product state resolution. The three-dimensional velocity distribution of state-selected reaction products is determined by ionizing the appropriate produnt, waiting for a delay while it recoils along the trajectory imparted by the reaction, and finally projecting the spatial distribution of ions onto a two dimensional screen using a puised electric field. Knowledge of the arrival time allows the ion position to be converted to a velocity, and the density of velocity projections can be inverted mathematically to provide the three-dimensional velocity distribution for the selected product. The main apparatus has been constructed and tested using photodissociations. The proposed research will both develop the new technique and employ it to investigate methyl radical, formyl radiral, and hydrogen atom reactions which are important in combustion processes. We intend specifically to characterize the reactions of $\mathrm{CH}_{3}$ with $\mathrm{H}_{2}$ and $\mathrm{H}_{2} \mathrm{CO}$; of $\mathrm{HCO}$ with $\mathrm{O}_{2}$; and of $\mathrm{H}$ with $\mathrm{CH}_{4}, \mathrm{CO}_{2}$, and $\mathrm{O}_{2}$.

\section{Notice}

This report was prepared as an account of work sponsored by the United States governinent. Neither the United States nor the Department of Energy, nor any of their employees, nor any of their contractors, subcontractors, or their employees, makes any warranty, express or implied, or assumes any legal liability or responsibility for the accuracy, completeness, or usefulness of ary information, apparatus, product or process disclosed or represents that its use would not infringe privately-owned rights. 


\section{Research Progress}

\section{Summery}

State-selected differential cross sections have been obtained by directly imaging the products of collisions in crossed molecular beams. The new technique allows final state resolution and simultaneous detection of all scattering angles. The method has been used to study inelastic collisions between $\mathrm{Ar}$ and $\left.\mathrm{NO}^{2} \mathrm{\Pi}_{1 / 2}, \mathrm{vm}=0, \mathrm{~J}=0.5\right)$ at a collision energy of $0.21 \mathrm{eV}$. Rotational rainbows in the product angular distribution are directly observed to change in position as a function of the final rotational state; the peak of the angular distribution moves toward the backward hemisphere and the angular distribution broadens with an increase in final rotational quantum number. The method relies on multiphoton ionization of the product, but is otherwise generally applicable to reactive as well as inelastic collisions.

\section{Progress}

State-resolved differential cross sections provide detailed information about the mechanism and potential energ'y surface goverring a chemical reaction, but they have heretofore been difficult to measure. This report describes our progress with a new technique for obtaining differential cross sections with both product etate resolution and simultaneous detection of all scattering angles. The three-dimensional velocity distribution of state-selected reaction products is determined by ionizing the appropriate product and accelerating the ions onto a detector using a pulsed electric field. The resulting image is a two-dimensional projection of the three-dimensional velocity distribution. Visual inspection provides immediate qualitative information about the reaction, while mathematical analysis can provide detailed differential cross sections. We have used the technique to investigate the inelastic collision process $A r+N O\left({ }^{2} \Pi_{1 / 2}, v=0, J=0.5\right) \rightarrow A r+N O\left({ }^{2} \Pi_{1 / 2}, u m 0, J^{\prime}\right)$ at a collision energy of $0.21 \mathrm{eV}$.

The collision dynamics of Ar + NO have been investigated previously at the total cross section level. ${ }^{3-3}$ Using laser-induced fluorescence and two crossed beams, Joswig et al. found interference 
effects in the scattering of $N O(u=0, J=0.5)$ by $\mathrm{He}, \mathrm{Ar}$, and $\mathrm{Ne}$; the total cross section for scattering to NO product states displayed oscillations as a function of the final rotational quantum number. The oscillations also appeared in coupled-states calculations using the electron gas potential of Nielson et al. The interference is due to scattering from the two different ends of the nearly homonuclear diatomic molecule. Two potential surfaces are important, one in which the orbital of the unpaired electron is in the plane containing the three atoms $\left(A^{\prime}\right)$, and one in which it is perpendicular to this plane (A). As shown by Alexander, ${ }^{\circ}$ multiplet changing collisions provide information ahout the difference between these two potentials, while multiplet conserving collisions provide information abqut the sum. Thus, state-resolved differential cross sections such as those made possible by the new method described here will be important in determining these two potential energy surfaces.

The new method for making these measurements is similar to that already used to determine the angular distribution of state-selected photof raginents. ${ }^{7 \cdot 12}$ A variant of the method has also been used to investigate the $\mathrm{H}+\mathrm{HI} \rightarrow \mathrm{H}_{2}+\mathrm{I}$ reaction, ${ }^{13}$ although not in crossed molecular beams. In our configuration, a doubly differentially pumped primary beam is formed by expanding $7 \%$ NO in He from a total pressure of 1100 torr through a pulsed nozzle $85 \mathrm{~cm}$ from the interaction region. The expansion yields a rotational distribution in the beam given by $N_{J=0.5}=1, N_{J}=1.5=0.037, N_{J}=2.5=0.006$. The secondary Ar beam is formed by expansion of 1040 torr through a pulsed nozzle $15 \mathrm{~cm}$ from the interaction region. A pulsed laser beam at a wavelength near $226 \mathrm{~nm}$ was propagated counter to the Ar beam and used to ionize state-selected NO products on the axis of a Wiley-McLaren ${ }^{14}$ time-offlight mass spectrometer. The ions were accelerated in a diroction perpendicular to the plane of the molecular beams by fields of $\sim 800$ volts $/ \mathrm{cm}$. After $\sim 6 \mu \mathrm{s}$, the ions struck the first of channelplate detectors, causing emission secondary electrons with a gain of $\sim 10^{7}$. Electrons from the channelplates were accelerated to a fast phosphor coated onto one end of a fiber-optic bundle mounted through a vacuum flange. A CID Camera (Xybion), gated according to the flight time for the mass of interest and equipped with a $480 \times 512$ pixel array, captured each image for averaging and 
analysis. A real-time video averager (Poynting RA-512) summed 256 images with 16 bit resolution. The most significant 8-bit slice of the resulting sum was normalized for laser power and stored in a computer. Alternate sets of images obtained with the Ar beam off were subtracted irom those with both beams on. Roughly 300,000 laser shots were averaged for Figures 2 and 3 shown below.

Figure 1 displays a superposition of two images obtained when a trace of NO is also seeded in the secondary Ar beam. In one of the two superimposed images the ionization laser propagates counter to the primary beam, while in the second it propagates counter to the secondary beam. The laser is tuned to ionize $\left.N O{ }^{2} \Pi_{1 / 2}, v=0, J=0.5\right)$, the principal component of each beam, so the illuminated section of each of the molecular beams is visible in the figure. The intersection of the two beams is

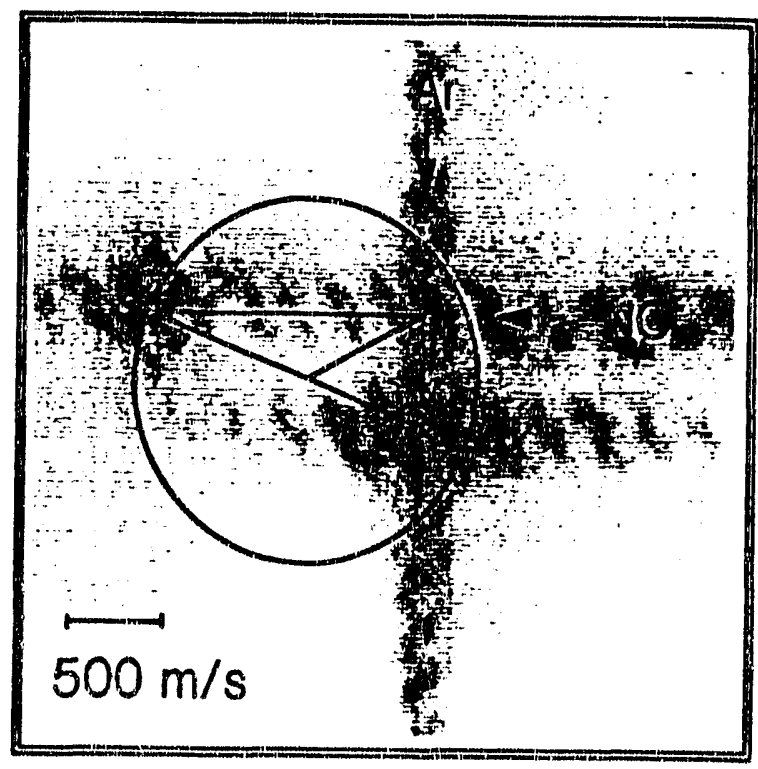
the collision region. The dark spots below and to the left of this intersection represent NO molecules that were ionized at the intersection of the beams and that traversed the intervening distance in the time between ionization and detection, $\sim 6 \mu \mathrm{s}$. Measurement of distances and the delay time provides the velocity, dispersion, and size for each molecular beam. A "Newton" diagram for the collision can be easily constructed for elastic collisions; it is superimposed on Fig. 1.

Figure 2 gives the image obtained when the probe laser (now propagating only counter to the pure Ar beam) is tuned to ionize $N O\left({ }^{2} \Pi_{1 / 2}, u=0, J=11.5\right)$. Despite some ionization of background room-temperature NO along the laser beam, the projection of the Newton sphere for the scattering is clearly visible. The scattering is actually symmetric about the relative velocity vector, but the detection sensitivity depends both on the velocity component perpendicular to the laser beam and on a center-of-mass to laboratory Jacobian. Qualitative analysis shows that the angular distribution is peaked at $30^{\circ}$ with a FWHM of about $20^{\circ}$. This rotational rainbow in the scattering angular 
distribution is expected for atom-diatom collisions, even at the level of classical mechanics and hard ellipse scattering. ${ }^{15}$

Figure 3 displays the image obtained when $N O\left({ }^{2} \Pi_{1 / 2}, v=0, J=18.5\right)$ is ionized. The product scattering is more diffuse and is centered much further toward the backward hemisphere; qualitative analysis places the peak at $60^{\circ}$ with a FWHM of $30^{\circ}$. In addition, the Newton sphere is measurably smaller, in accord with the lesser available energy

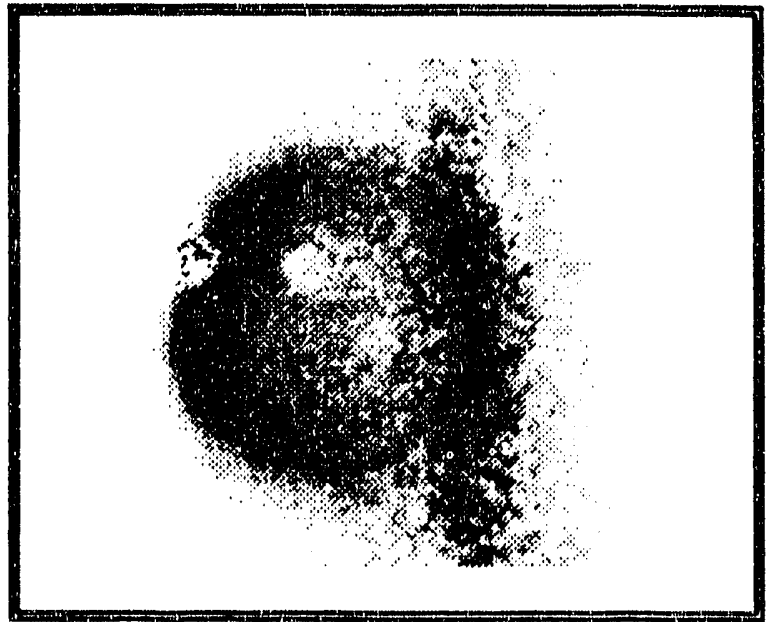

Figure 2 Two-dimensional projection of the scattering distribution for $\left.N O{ }^{2} \Pi_{1 / 2}, v=0, J=11.5\right)$.

for translation. Images were also recorded for $\mathrm{J}=9.5\left(0 \pm 10^{\circ}, \mathrm{FWHM}=20^{\circ}\right)$ and $\mathrm{J}=15.5\left(40^{\circ}\right.$, FWHM=30 $0^{\circ}$.

The figures show that the imaging technique can provide direct qualitative information about the collision dynamics. Although a quantitative analysis of the images is not yet complete, it is clear to us that the detailed differential cross section can be obtained by a forward convolution technique which includes the density to flux transformation and the center-of-mass to laboratory Jacobian. The advantages of the technique are: 1) that the state-resolution is limited only by the spectral resolution of the

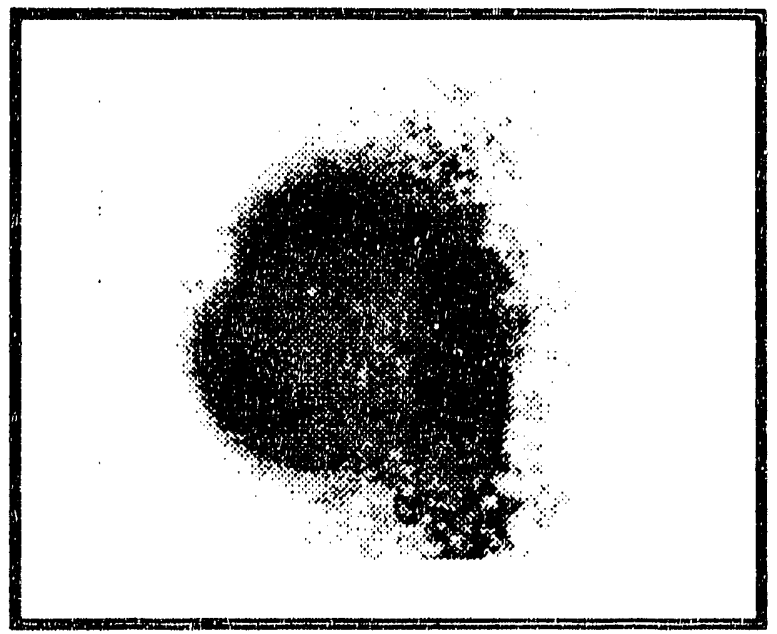

FIgure 3 Two-dimensional projection of the scattering distribution for $N O{ }^{2} \Pi_{1 / 3}, v=0, J=18.5$ ). laser and the energy separation between molecular eigenstates, and 2) that the entire angular distribution is recorded for each laser pulse. Potential disadvantages of the technique are that the molecule to be probed must be able to undergo state-selected multiphoton ionization. The data thus 
far provide encouragement that the state-selected differential cross sections needed to determine the two potential energy surfaces involved in the Ar+NO system can be obtained.

\section{Planned Work for Year Two of the Grant Perlod}

Future research will both develop further the new technique and employ it to investigate methyl radical, formyl radical, and hydrogen atom reactions which are important in combustion processes. We intend specifically to characterize the reactions of $\mathrm{CH}_{3}$ with $\mathrm{H}_{2}$ and $\mathrm{H}_{2} \mathrm{CO}$; of $\mathrm{HCO}$ with $\mathrm{O}_{2}$; and of $\mathrm{H}$ with $\mathrm{CH}_{4}, \mathrm{CO}_{2}$, and $\mathrm{O}_{2}$. The reaction we will probably start with is $\mathrm{H}+\mathrm{O}_{2}$. The first step in the process will be to create (and detect) a beam of $\mathrm{H}$ atoms, formed by photolysis of $\mathrm{H}_{2} \mathrm{~S}$. About $2.4 \times 10^{-4}$ torr of $\mathrm{H}$ atoms can be delivered at the interaction region with the molecular beam, as outlined in our original proposal. We will start by ionizing the $\mathbf{H}$ atoms in this region so as to have a method for optimization of the source beam. Ionization will be achieved by ionization after excitation of the Lyman alpha transition. 


\section{References}

1 A. S. Sudbo and M. M. T. Loy, Chem. Phys. Lett. 82, 135 (1981).

2 P. Andresen, H. Joswig, H. Pauly, and R. Schinke, J. Chem. Phys. 77, 2204 (1982).

S H. Joswig, P. Andresen, and R. Schinke, J. Chem. Phys. 85, 1904 (1986).

- G. C. Nielson, S. A. Parker, and R. T Pack, J. Chem. Phys. 66, 1396 (1977).

C. W. McCurdy and W. H. Miller, J. Chem. Phys. 67, 463 (1977).

B M. H. Alexander, J. Chem. Phys. 76, 5974 (1982).

- 7 D. W. Chandler and P. L. Houston, J. Chem. Phys. 871445 (1987).

8 D. W. Chandler, J. W. Thoman, Jr., M H. M. Janssen, and D. H. Parker, Chem. Phys. Lett. 156151 (1989).

- D. W. Chandler, M. H. M. Janssen, S. Stolte, R. N. Strickland, J. W. Thoman, Jr., and D. H. Parker, J. Phys. Chem. 944839 (1990).

10 J. W. Thoman, D. W. Chandler, D. H. Parker, M. H. M. Janssen, Laser Chem. 927 (1988).

11 D. P. Baldwin, M. A. Buntine, and D. W. Chandler, J. Chem. Phys. 936578 (1990).

12 T. Suzuki, V. P. Hradil, S. A. Hewitt, P. L. Houston, and B. J. Whitaker, Chem. Phys. Lett. 187, 257 (1991).

13 M. A. Buntine, D. P. Baldwin, R. N. Zare, and D. W. Chandler, J. Chem. Phys. 944672 (1991).

14 W. C. Wiley and 1. H. McLaren, Rev. Sci. Instrum. 26 (1955) 1150.

16 R. Schinke and J. M. Bowman, "Rotational Rainbows in Atom-Diatom Scattering," in Molecular Collision Dynamics, Chapter 4, J. M. Bowman, ed., (Springer-Verlag, Berlin, 1983), p. 61. 

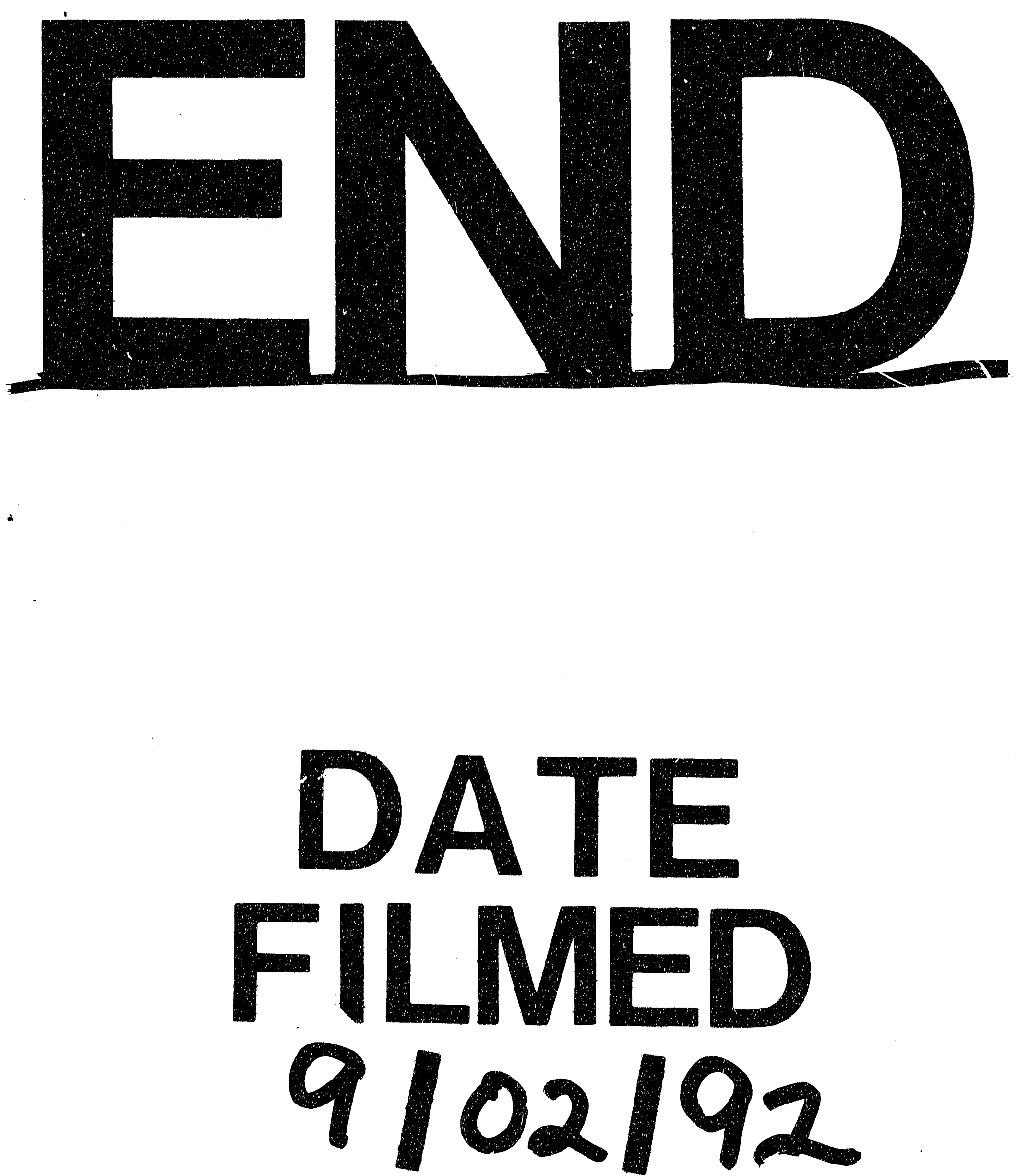
